\title{
Substitutional oxygen activated photoluminescence enhancement in monolayer transition metal dichalcogenides
}

\author{
Shilong Zhao ${ }^{1 \dagger}$, Junyang Tan ${ }^{1,2 \dagger}$, Chengxuan $\mathrm{Ke}^{3 \dagger}$, Simin Feng ${ }^{1}$, Yongjue Lai ${ }^{1}$, Baofu Ding ${ }^{1}$, \\ Guangfu Luo ${ }^{3,4^{*}}$, Junhao $\operatorname{Lin}^{2,5^{*}}$ and Bilu Liu ${ }^{*}$
}

\begin{abstract}
Atomically thin transition metal dichalcogenides (TMDCs) are intriguing semiconductors for photonics and optoelectronics, and therefore enhancing their photoluminescence $(\mathrm{PL})$ efficiency is crucial for these applications. Many efforts have been contributed to enhancing the PL performance of monolayer TMDCs, yet the complexity between the microstructure and the PL efficiency has hindered the manipulation of their PL properties. Here we demonstrate that the PL intensity of the monolayer TMDC can be enhanced by nearly one order of magnitude with a $\sim 20 \%$ narrower spectral linewidth after a pre-activation plateau using laser irradiation in ambient environment. Combined experimental and theoretical studies reveal that low-power laser irradiation generates many sulfur vacancy clusters, which are subsequently filled up by oxygen, and the lattice substitutional oxygen clusters induce the dramatic PL enhancement of monolayer $\mathrm{WS}_{2}$. Such PL enhancement phenomenon is found to be universal for other monolayer TMDCs, and thus would benefit their versatile optical applications.
\end{abstract}

Keywords: two-dimensional materials, transition metal dichalcogenides, photoluminescence enhancement, laser, oxygen

\section{INTRODUCTION}

Two-dimensional (2D) semiconducting transition metal dichalcogenides (TMDCs) with the formula of $\mathrm{MX}_{2}$ ( $\mathrm{M}$ for transition metals and $\mathrm{X}$ for chalcogenides, such as $\mathrm{S}$, Se, or Te) are promising candidates for photonic or optoelectronic applications due to their direct bandgaps ranging from near-infrared to visible light $[1-5]$, strong light-matter interactions $[3,6]$, valley polarization [7-9], etc. One important characteristic for optical applications is the excitonic photoluminescence (PL), which is strongly associated with atomic level structures of materials [10,11]. Many efforts have been devoted to improving the PL performance of monolayer TMDCs through defect engineering, while based on somehow two opposite strategies $[1,12-27]$. The first strategy is to make TMDCs close to intrinsic state by reducing or passivating defects [12-14], electrostatic gating [15], or surface engineering [18]. This strategy enhances the PL performances of TMDCs by strongly suppressing the non-radiative recombination pathway caused by the structural distortions. The second strategy is making the structures of TMDCs more defective to enhance their PL performances [1,16,19-21,23-25,27]. For example, $\mathrm{He}$ et al. [24] reported increased PL intensity of monolayer $\mathrm{WS}_{2}$ when oxidized in ambient conditions. Tongay et al. [16] reported the generation of anion vacancy in exfoliated TMDCs, which is believed to be an effective method to manipulate their PL intensities. In another study, Nan et al. [23] reported increased PL intensity at the crack region of monolayer $\mathrm{MoS}_{2}$. So far, the inconsistency between these two strategies indicates complicated structure-PL relationship in such monolayer semiconductors. Therefore, bridging the gap between the macroscopic PL performance and the microscopic structural perturbations (e.g., point defects and substitutional impurities) is essential for understanding the PL property of monolayer TMDCs.

Here we observed an anomalous PL enhancement of monolayer $\mathrm{WS}_{2}$ by continuous low-power laser irradiation. The PL intensity of monolayer $\mathrm{WS}_{2}$ increases by nearly one order of magnitude, and the spectral linewidth decreases by $\sim 20 \%$. Interestingly, such PL enhancement also accompanies a preactivation plateau in which the PL remains almost unchanged. Scanning transmission electron microscopy (STEM) results show that the increased formation of sulfur vacancies during the pre-activation plateau induced by laser irradiation plays an important role in activating the PL enhancement process. Density functional theory (DFT) calculations further reveal that the laser-induced sulfur vacancy clusters can be filled up by oxygen, and elaborate that these oxygen substitutional defect clusters dramatically increase the emission energy of defective $\mathrm{WS}_{2}$ at a critical concentration. We have also observed similar

\footnotetext{
${ }^{1}$ Shenzhen Geim Graphene Center, Tsinghua-Berkeley Shenzhen Institute and Institute of Materials Research, Tsinghua Shenzhen International Graduate School, Tsinghua University, Shenzhen 518055, China

${ }^{2}$ Department of Physics, Southern University of Science and Technology, Shenzhen 518055, China

${ }^{3}$ Department of Materials Science and Engineering, Southern University of Science and Technology, Shenzhen 518055, China

${ }^{4}$ Guangdong Provincial Key Laboratory of Computational Science and Material Design, Southern University of Science and Technology, Shenzhen 518055, China

${ }^{5}$ Shenzhen Key Laboratory of Advanced Quantum Functional Materials and Devices, Southern University of Science and Technology, Shenzhen 518055, China

† These authors contributed equally to this work.

* Corresponding authors (emails: luogf@sustech.edu.cn (Luo G); linjh@sustech.edu.cn (Lin J); bilu.liu@sz.tsinghua.edu.cn (Liu B))
} 
anomalous PL enhancement phenomena (plateau and sudden increase of $\mathrm{PL}$ ) in other TMDC monolayer materials such as $\mathrm{WSe}_{2}, \mathrm{MoS}_{2}$, and $\mathrm{MoSe}_{2}$, verifying the universality of substitutional oxygen in the PL enhancement process.

\section{EXPERIMENTAL SECTION}

\section{Sample preparation}

Monolayer TMDCs $\left(\mathrm{MoS}_{2}, \mathrm{MoSe}_{2}, \mathrm{WS}_{2}\right.$, and $\left.\mathrm{WSe}_{2}\right)$ were mechanically exfoliated from their bulk crystals $(2 \mathrm{H}$ phase, HQ graphene) onto the surface of $285 \mathrm{~nm} \mathrm{SiO} 2 / \mathrm{Si}$ substrates. Monolayer $\mathrm{WS}_{2}$ was transferred onto the TEM grid for characterization using a dry-transfer method with a polyethylene terephthalate (PET) stamp [28]. Briefly, a PET stamp was used to pick up the as-exfoliated monolayer $\mathrm{WS}_{2}$ and put in contact with a Ted Pella Quantifoil TEM grid (657-200-AU, 2 um holes) at $60^{\circ} \mathrm{C}$. The PET was then removed by immersing the stamp and TEM grid in dichloromethane overnight.

\section{Optical measurements}

As an excitation source, a $532-\mathrm{nm}(2.33 \mathrm{eV})$ laser beam was focused on monolayer TMDCs with a spot diameter of $\sim 1 \mu \mathrm{m}$. The PL and Raman spectra of monolayer TMDCs were collected by a Horiba confocal Raman spectrometer (LabRAM HR Evolution) in reflection geometry. For time-dependent PL measurements, the integrated time for each spectrum was set to be $0.1 \mathrm{~s}$ unless otherwise claimed and the time intervals for two spectra were also set to be $0.1 \mathrm{~s}$ (the minimum value our setup can provide). The laser power was set to be $2 \mu \mathrm{W}$ unless otherwise claimed. The PL mappings of monolayer TMDCs were done before and after high-temperature thermal annealing at $\sim 300^{\circ} \mathrm{C}$ in air.

\section{Data handling}

The PL spectrum of monolayer $\mathrm{WS}_{2}$ was fitted using two Voigt functions based on the consideration of exciton and trion components. The resultant parameters were used as initial guess parameters for the batch peak fitting of the time-dependent PL spectra. The fitting tolerance was less than $10^{-6}$. The deconvoluted exciton and trion spectra were displayed in a $2 \mathrm{D}$ color plot manner to show their evolution under laser irradiation.

\section{Atomic-resolution STEM measurements}

Atomic-resolution high-angle annular dark field STEM (HAADF-STEM) images were acquired with a double-aberration-corrected STEM (Titan Cubed Themis G2 300) operated at $60 \mathrm{kV}$. The semi-convergence angle was about $30 \mathrm{mrad}$ and the semi-collection range of the STEM image was around 54168 mrad.

\section{First principles calculations}

All calculations were performed using the DFT as implemented in the Vienna ab-initio Simulation Package [29]. The exchangecorrelation functional was chosen in the form of generalized gradient approximation, as parameterized by Perdew, Burke, and Ernzerhof [30]. The projector augmented wave [31,32] pseudopotentials of $\mathrm{W}\left(6 \mathrm{~s}^{2} 5 \mathrm{~d}^{4}\right)$ for W, S_GW $\left(3 \mathrm{~s}^{2} 3 \mathrm{p}^{4}\right)$ for S, O_GW_new $\left(2 s^{2} 2 p^{4}\right)$ for $O$ were used, together with a plane wave cutoff of 310 and $521 \mathrm{eV}$ for systems without and with oxygen, respectively. The supercell size was $5 \times 5 \times 1$ of the primitive cells of $\mathrm{WS}_{2}$ with $a=b=15.89 \AA$, and the Brillouin zone was sampled with a $12 \times 12 \times 1 \Gamma$-centered mesh.

\section{RESULTS AND DISCUSSION}

We first exfoliated monolayer TMDCs (2H phase) using the Scotch-tape method and studied their PL property upon laser irradiation (Fig. 1a, see the EXPERIMENTAL SECTION for details). Taking monolayer $\mathrm{WS}_{2}$ as an example, it was mechanically exfoliated onto silicon substrates with a $285-\mathrm{nm}$ -thick oxide layer. The monolayer flake was identified by optical microscopy image contrasts and further confirmed by PL spectra, as shown in Fig. S1 in the Supplementary information. The as-exfoliated monolayer $\mathrm{WS}_{2}$ was continuously irradiated by laser in air and its PL spectra were continuously monitored (see details in the EXPERIMENTAL SECTION). A representative record of the PL spectrum evolution is shown in Fig. $1 \mathrm{~b}$ in a $2 \mathrm{D}$ colorplot form. The color scale corresponds to PL intensity, a horizontal line traces the PL intensity changes at the given emission energy, and the vertical line cut represents the PL spectrum at the given time. As an example, Fig. 1c shows how the peak intensity in the PL spectrum of monolayer $\mathrm{WS}_{2}$ evolves along the black dashed lines (Fig. 1b) at $\sim 1.99 \mathrm{eV}$. It clearly shows that the exposure of monolayer $\mathrm{WS}_{2}$ to low-power laser irradiation slightly increases the PL intensity to a steady state, which then remains in the same plateau for $\sim 80 \mathrm{~s}$, similar to the results in previous reports [17,33]. Strikingly, we observed a sudden intensity increase starting at $\sim 88 \mathrm{~s}$, which has not been reported. This anomalous sharp increase in PL intensity reaches its peak value at $\sim 91 \mathrm{~s}$. The inset in Fig. 1c further compares the PL spectra of monolayer $\mathrm{WS}_{2}$ recorded at $0 \mathrm{~s}$ (blue line) and $91 \mathrm{~s}$ (red line). The peak intensity at $91 \mathrm{~s}$ is $\sim 8$ times higher and the peak position redshifts by $10 \mathrm{meV}$ in comparison with the initial PL spectra at $0 \mathrm{~s}$. The redshift of the peak position possibly suggests the formation of laser-induced defective states near the band edge of $\mathrm{WS}_{2}$. The PL intensity was followed by a subsequent decrease due to obvious damage of the sample.

The PL spectra of monolayer $\mathrm{WS}_{2}$ can be well fitted using two Voigt functions based on the consideration of exciton and trion components [34], as shown in Fig. S2. The resultant evolution of decomposed exciton and trion components are summarized in Fig. S3. Both the intensities of exciton (Fig. S3a) and trion components (Fig. S3b) reach a maximum at $91 \mathrm{~s}$, showing a similar enhancement feature to the total PL spectra. In addition, the linewidth of the exciton component (red line, Fig. 1d) increases slightly when the irradiation time elongates and suddenly drops by $\sim 20 \%$ at $\sim 91 \mathrm{~s}$ when the PL peak intensity reaches a maximum. Similarly, the peak position of exciton (blue line, Fig. 1d) redshifts slowly under laser irradiation and blueshifts slightly at $\sim 91 \mathrm{~s}$. However, trion shows a different behavior (Fig. 1e). The linewidth of trion keeps almost unchanged and the peak position blueshifts by $\sim 10 \mathrm{meV}$ when the PL peak intensity reaches a maximum. These observations indicate that the laser irradiation induces defective states before the PL enhancement and suppresses these defective states thereafter. Overall, our results show that continuous laser irradiation can modulate the $\mathrm{PL}$ performance of monolayer $\mathrm{WS}_{2}$.

To shed light on the origin of the anomalous PL intensity change of $\mathrm{WS}_{2}$, we monitored its structural alternation at atomic level using aberration-corrected HAADF-STEM at five time nodes labeled as b-f during stages I to III in Fig. 2a (reproduced from Fig. 1c). The corresponding results are shown in Fig. 2b-f, where single $\left(\mathrm{V}_{1 \mathrm{~S}}\right)$ and double sulfur vacancies $\left(\mathrm{V}_{2 \mathrm{~S}}\right)$ are marked 

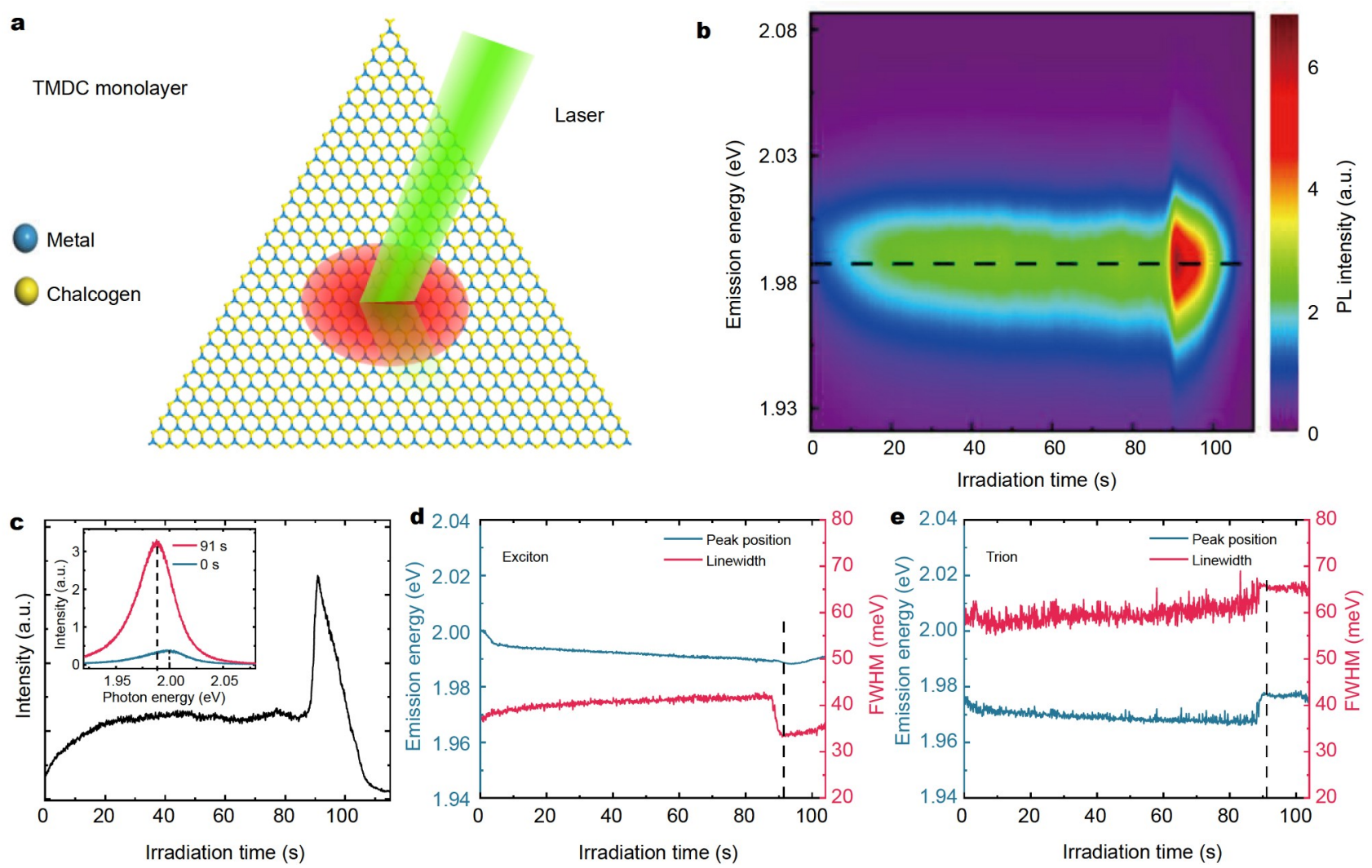

Figure 1 PL enhancement of monolayer TMDC under laser irradiation. (a) A schematic illustration of the PL enhancement for monolayer TMDC under laser irradiation. (b) Time-dependent PL spectrum of monolayer $\mathrm{WS}_{2}$ under continuous laser irradiation. The PL intensity evolution along the black dashed line is shown in (c). (c) PL peak $(\sim 1.99 \mathrm{eV})$ intensity evolution of monolayer $\mathrm{WS}_{2}$ under continuous laser irradiation. Inset: two representative PL spectra at different irradiation times: blue for $0 \mathrm{~s}$ and red for $91 \mathrm{~s}$. (d, e) The spectral linewidth (red) and peak position (blue) evolution of exciton component (d) and trion component (e) under continuous laser irradiation. The dashed lines in (d) and (e) label the time when PL intensity reaches a maximum.

by yellow and red circles, respectively. Fig. $2 \mathrm{~b}$ shows that monolayer $\mathrm{WS}_{2}$ has $\sim 1.6 \% \mathrm{~V}_{1 \mathrm{~s}}$ and $\sim 2.0 \% \mathrm{~V}_{2 \mathrm{~S}}$ defects at its pristine stage (Stage I). No significant change of defect density is observed at $\mathrm{b}\left(\sim 1.6 \% \mathrm{~V}_{1 \mathrm{~S}}\right)$ and $\mathrm{c}\left(\sim 2.1 \% \mathrm{~V}_{1 \mathrm{~S}}\right)$, indicating that the PL enhancement in Stage I is irrelevant with the defect density, which possibly originates from the removal of adsorbed contaminations from the surface of monolayer $\mathrm{WS}_{2}$ by laser heating. In the steady pre-activation stage (Stage II), the defect density of $\mathrm{V}_{1 \mathrm{~S}}$ increased to $4.9 \%$ at point $\mathrm{d}$ (Fig. $2 \mathrm{~d}$ ), and further to $6.2 \%$ at point e (Fig. 2e). Similar increase is also seen for $\mathrm{V}_{2 \mathrm{~S}}$, from $1.9 \%$ to $5.6 \%$ as irradiation time increases, which is summarized in Fig. 2g. Overall, during Stage II, the density of sulfur vacancies gradually rises with irradiation time, while the linewidths (peak position) of both exciton and trion increase (redshift) slowly, indicating that laser-induced sulfur vacancies modulate the PL performance of $\mathrm{WS}_{2}$, but have a trivial contribution in the enhancement of PL intensity. On the other hand, during/after the activation of the sharp increase of PL (Stage III), we find that further laser irradiation may dramatically increase the density of sulfur vacancies. This is evidenced in point $f$, where the generation of many sulfur vacancies finally results in the formation of holes with the diameter ranging from several to tens of nanometers, as shown in Fig. 2f. These holes grow to a much larger size (even to $\sim 80 \mathrm{~nm}$ as shown in Fig. S4) at the end of the Stage III, and lead to a significant drop in the effective emission areas and the resultant decrease of the PL intensity. The spectrum features of both exciton and trion show little variation after the PL intensity reaches a maximum, as indicated by the black dashed lines in Fig. 1d, e, it is reasonable to investigate the representative structural feature of stage III at point $f$.

The spectral feature of exciton (and trion) shows different trends before and after the PL enhancement process (Fig. 1d, e), indicating the evolution of sulfur vacancies to nano-sized holes is the key factor to understand the sharp PL enhancement process. Fig. 3a-c show characteristic HAADF-STEM images of monolayer $\mathrm{WS}_{2}$ at stage III, where nano-sized holes with different diameters ranging from $0.8-80 \mathrm{~nm}$ are identified clearly (for more details, see Fig. S4). Small holes with $\sim 1 \mathrm{~nm}$ size usually have $\mathrm{W}$-terminated edges, as shown in Fig. 3a, which are related to the loss of $\mathrm{WS}_{6}$ cluster possibly through multiple double-sulfur vacancies nearby [35]. Fig. 3b, c show large holes with diameters of $\sim 2$ and $\sim 30 \mathrm{~nm}$ (see Figs S5 and S6 for more information), whose edges are also mainly terminated by $\mathrm{W}$ atoms. At the hole edges, particles with brighter contrast than tungsten atoms are often observed, as guided by the blue arrows in Fig. 3a-c (see Fig. S5). The chemical composition of these brighter clusters is determined to be tungsten oxides by electron energy loss spectroscopy (EELS), where the characteristic onset of the oxygen $\mathrm{K}$ edge can be clearly distinguished as shown in Fig. 3d. The existence of tungsten oxides in irradiated $\mathrm{WS}_{2}$ is further verified by energy dispersive X-ray spectroscopy (EDX) mappings as shown in Fig. 3e, $\mathrm{f}$, where the spatial distribution of $\mathrm{O}, \mathrm{S}$, and $\mathrm{W}$ elements is marked by red, green, and blue colors, respectively. The $\mathrm{S}$ and $\mathrm{W}$ elements are evenly distributed in the 

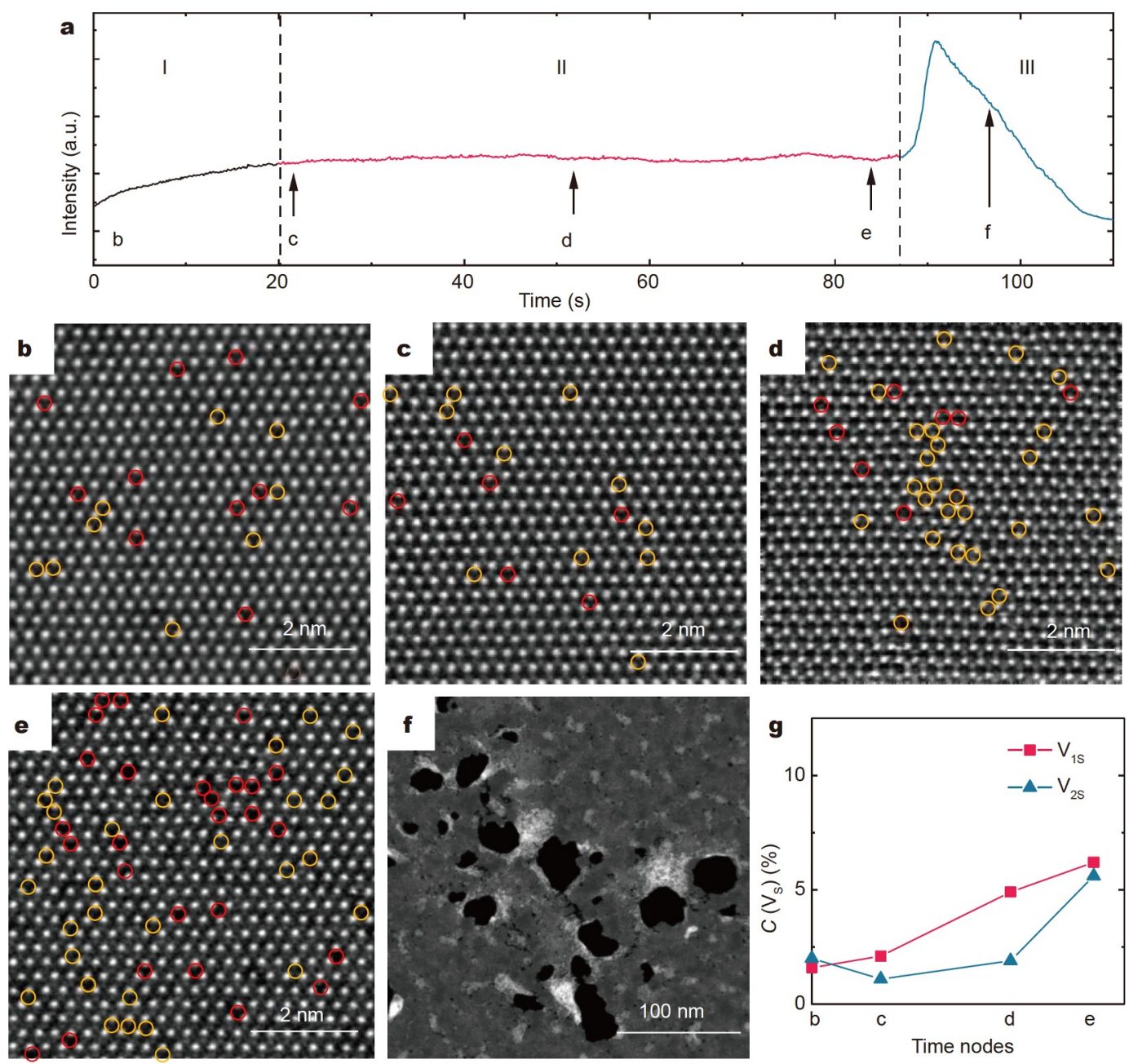

Figure 2 Laser-induced structural variation of monolayer $\mathrm{WS}_{2}$ upon time increasing. (a) The irradiation time-dependent PL peak intensity of monolayer $\mathrm{WS}_{2}$. Three stages can be identified as Stage I, II, and III. (b-f) HAADF-STEM images corresponding to different irradiation times as labeled in (a), yellow and red circles indicate the single and double sulfur vacancies, respectively. $(\mathrm{g})$ The sulfur vacancy concentration $\left(C\left(\mathrm{~V}_{\mathrm{s}}\right)\right)$ at different time nodes, as labeled in (a).

undamaged area of $\mathrm{WS}_{2}$, while oxygen element is distributed in the whole area of $\mathrm{WS}_{2}$ and accumulated near the edges of holes, overlapping with the bright cluster region. The formation of the holes suggests a metastable sulfur vacancy agglomeration before structural breaking, while the vacancy bonded oxygen indicates the oxygen molecule in air may further involve in the vacancy agglomeration and lattice reactions within $\mathrm{WS}_{2}$. The observation of holes and bonded oxygen provides hints to understand the sharp activation of the PL enhancement process.

To reveal the underlying mechanism of the laser-induced PL modulation, we carried out first-principles calculations based on DFT to examine the electronic properties of $\mathrm{WS}_{2}$ with and without defects. Fig. 4 a shows the representative sulfur vacancy $\mathrm{V}_{n \mathrm{~S}}$ ( $n$ denotes the number of sulfur atoms) and the oxygensubstituted sulfur $\mathrm{O}_{n \mathrm{~S}}$, both of which are major defects as suggested by the HADDF-STEM images in Figs 2 and 3. In Stages I and II, the temperature of $\mathrm{WS}_{2}$ is expected to be relatively low and only a limited number of $\mathrm{V}_{\mathrm{S}}$ are formed. Fig. $4 \mathrm{~b}$ shows that $\mathrm{V}_{\mathrm{S}}$ exhibits PL peaks significantly $(>0.5 \mathrm{eV})$ lower than the pristine one. For instance, $\mathrm{V}_{6 \mathrm{~S}}$ shows a PL peak about $1.3 \mathrm{eV}$ below the pristine PL peak, which is not captured in our PL spectrum. The low defect concentration and low PL peak posi- tion explain the fact that the PL spectra remain nearly unchanged in the observed energy region in Stages I and II.

As heat accumulates at the $\mathrm{WS}_{2} / \mathrm{SiO}_{2}$ interface during the continuous laser irradiation on monolayer $\mathrm{WS}_{2}$ due to the low heat dissipation rate of silicon dioxide [36], the temperature of $\mathrm{WS}_{2}$ is expected to increase accordingly with time. At the beginning of the activated PL enhancement (Stage III), the temperature of $\mathrm{WS}_{2}$ is supposed to reach a critical value at which the thermal energy is comparable to the formation energy of sulfur vacancy. Further increase of the temperature results in a nearly exponential increase of $\mathrm{V}_{\mathrm{S}}$ concentration as schematically shown in Fig. 4c. Since filling chalcogen vacancies with oxygen atoms is thermodynamically preferable [37], the dramatic increase of sulfur vacancies will lead to a dramatic formation of $\mathrm{O}_{n \mathrm{~s}}$, even clusters (e.g., $\mathrm{O}_{6 \mathrm{~s}}$ ). Fig. $4 \mathrm{~b}$ shows that $\mathrm{O}_{n \mathrm{~s}}$ exhibits PL peak about $0.3 \mathrm{eV}$ below the pristine PL peak. Because a lower PL peak corresponds to a smaller bandgap and thus more excited electrons, the PL intensity of $\mathrm{O}_{n \mathrm{~S}}$ is also envisioned to increase dramatically. These explain qualitatively the red-shifted PL peak positions and the dramatic increase of PL intensity as observed in Fig. 1c. We also performed calculations on the electronic structures of nano-sized holes with S- or O-termi- 

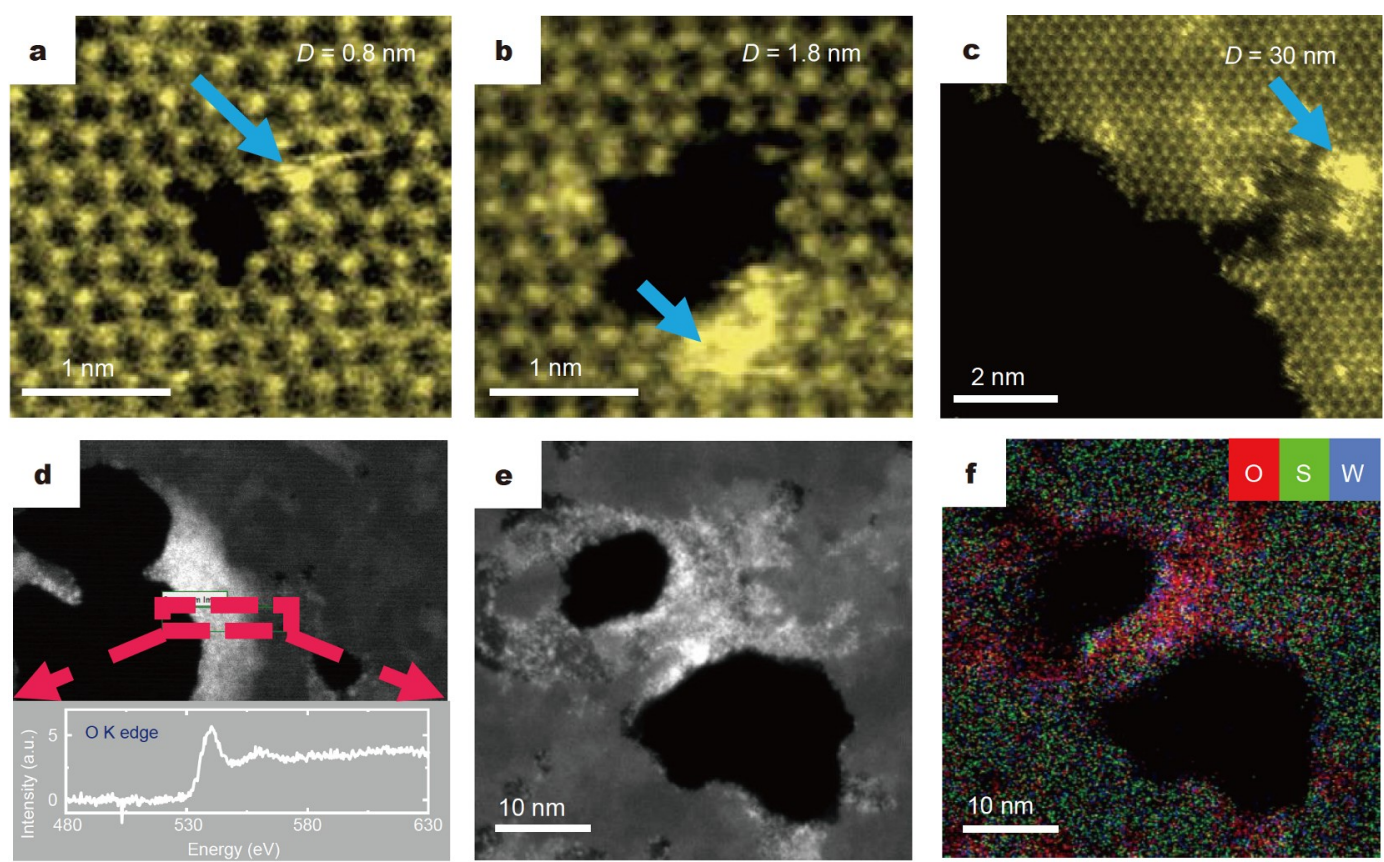

Figure 3 The structure of the nano-sized holes in $\mathrm{WS}_{2}$ formed during the Stage III. (a-c) Representative HAADF-STEM images of monolayer WS 2 after laser irradiation, of which holes have different diameters (labeled as $D$ ). The blue arrows indicate the particles coming from the holes during laser irradiation. The edges of these holes are predominately composed of W atoms (bright). (d) HAADF-STEM image of the edge of holes. Inset: EELS spectrum shows clearly the $K$ edge for oxygen, demonstrating the existence of tungsten oxides in bright particles. (e, f) HAADF-STEM (e) and corresponding EDX mapping images (f) of laser-irradiated $\mathrm{WS}_{2}$. The red, green, and blue in ( $\mathrm{f}$ ) indicate the EDX mapping of $\mathrm{O}, \mathrm{S}$, and $\mathrm{W}$, respectively.
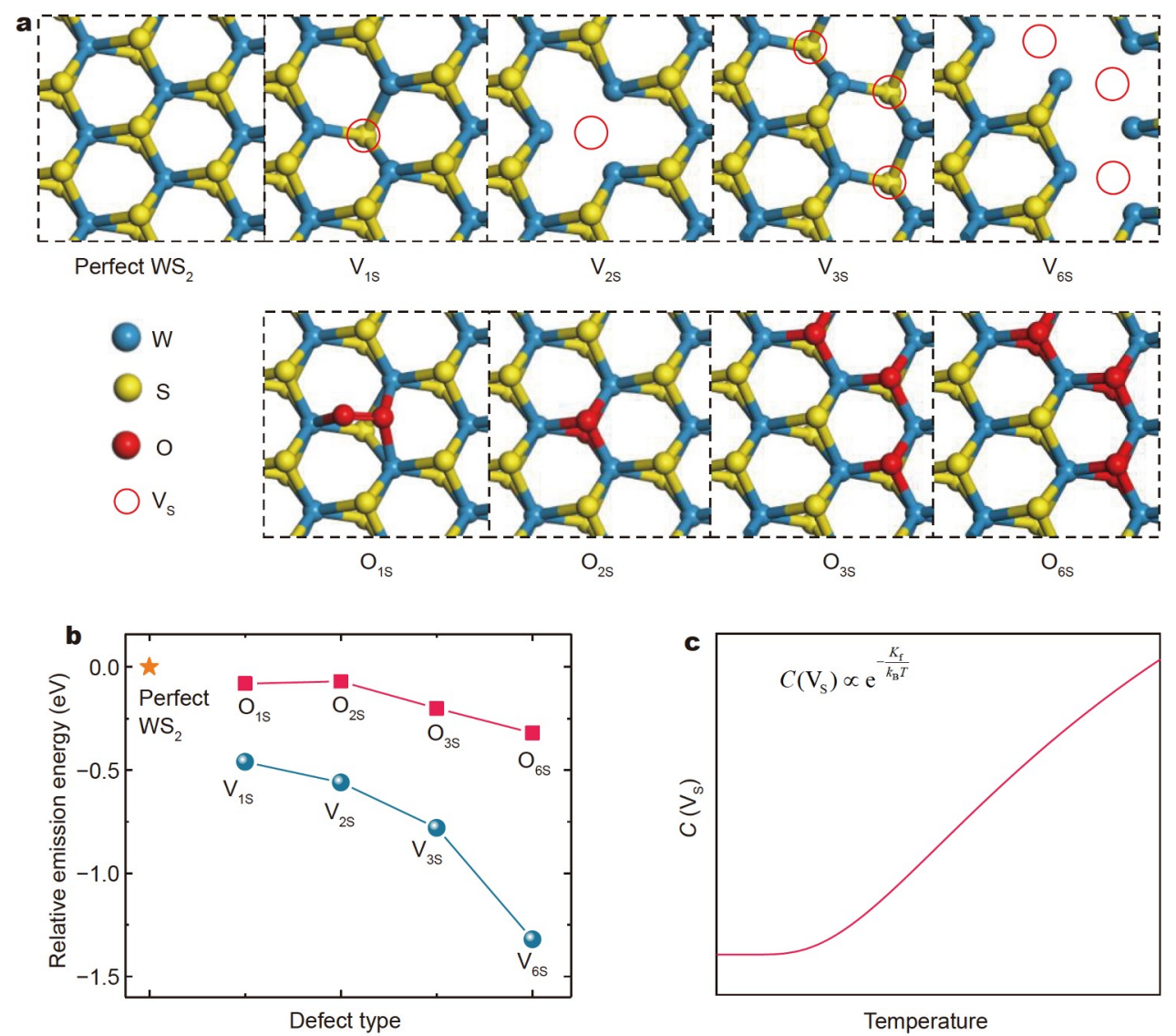

Figure 4 Theoretical interpretation for laser-induced PL modulation of monolayer WS $\mathrm{W}_{2}$. (a) Structural models of a perfect and several types of defective monolayer $\mathrm{WS}_{2}$. (b) Dependence of relative PL peak position on defect types. (c) Schematic relationship between $\mathrm{V}_{\mathrm{S}}$ concentration and temperature. 

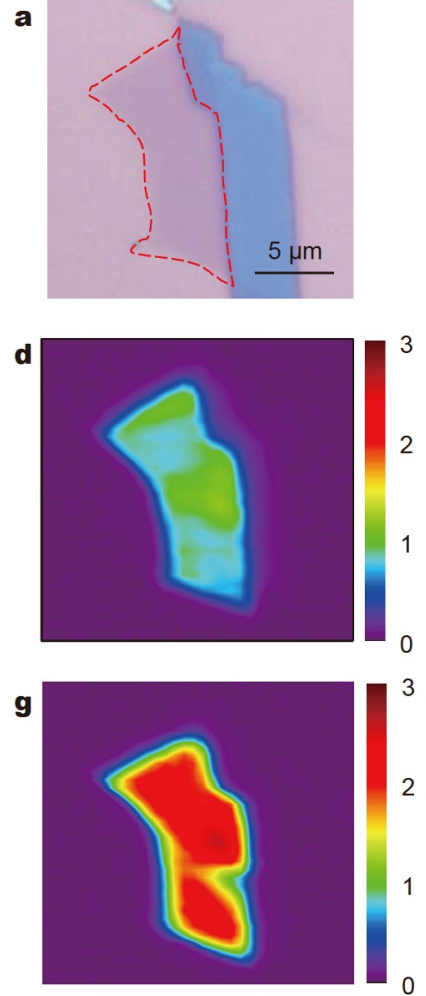

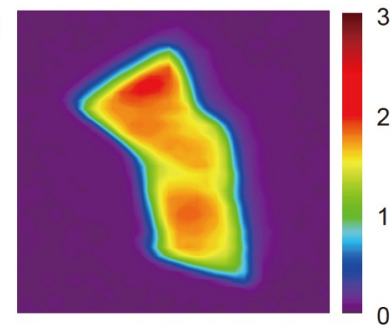

e

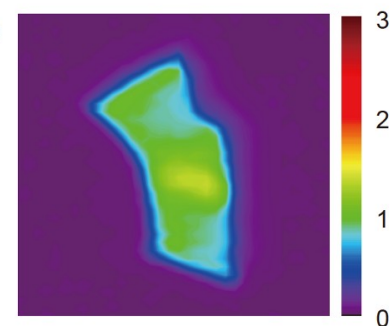

h

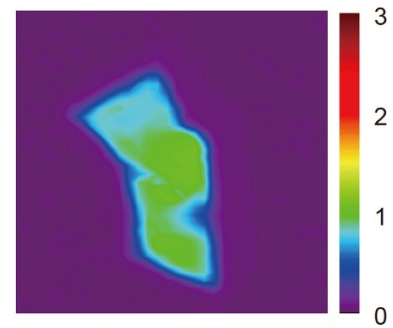

c

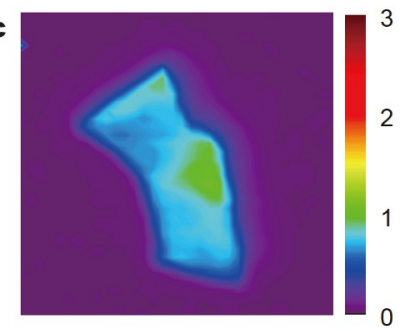

$f$
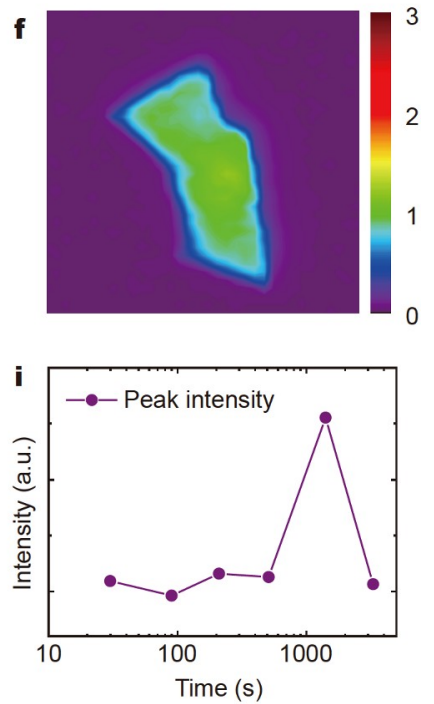

Figure 5 Oxidization-induced PL enhancement of monolayer $\mathrm{WS}_{2}$. (a) The optical microscopy image of exfoliated $\mathrm{WS}_{2}$ monolayers on a SiO $\mathrm{m}_{2} / \mathrm{Si}$ substrate. (b-h) PL intensity mappings of as-exfoliated $\mathrm{WS}_{2}$ flakes after heating on a hot plate for different times (b: 0 min, $\mathrm{c}: 0.5 \mathrm{~min}$, d: $1.5 \mathrm{~min}$, e: $3.5 \mathrm{~min}, \mathrm{f}: 8.5 \mathrm{~min}$, g: $23.5 \mathrm{~min}$, h: $55 \mathrm{~min}$ ). (i) The PL peak intensity changes when heated for different time periods. It could be observed that the PL intensity increases to the maximum value followed by a decrease of intensity.

nated edges, as shown in Fig. S7. These nano-sized holes with Sor O-terminated edges show much lower emission energy than that of pristine $\mathrm{WS}_{2}$, which indicates that they did not contribute strongly to the PL enhancement process. In the latter part of stage III, the elevated temperature of $\mathrm{WS}_{2}$ is supposed to induce excessive defects that boost the formation of nano-sized holes (Fig. 2f), leading to a sharp decrease of PL intensity (Fig. 1c).

The proposed mechanism and its generality were further verified by our controlled experiments in $\mathrm{N}_{2}$ environment and the thermal annealing of $\mathrm{WS}_{2}$ and several other TMDCs in air. The low-power laser irradiation of monolayer $\mathrm{WS}_{2}$ in a sealed chamber filled with $\mathrm{N}_{2}$ did not show significant PL enhancement even for long time irradiation (see Fig. S8), indicating a significant role of oxygen in the PL enhancement. Fig. 5a shows an optical microscopy image of monolayer $\mathrm{WS}_{2}$ that was thermally annealed at $300^{\circ} \mathrm{C}$ for a certain period of time. The corresponding PL intensity mapping results are shown in Fig. 5b-h. Fig. $5 \mathrm{i}$ traces the PL intensity changes in Fig. 5c-h, demonstrating a similar PL emission activation trend (after a preactivation plateau then sudden increase). We found that the oxidization-induced PL enhancement was universal for four typical monolayer TMDCs $\left(\mathrm{MoS}_{2}, \mathrm{WS}_{2}, \mathrm{MoSe}_{2}\right.$, and $\left.\mathrm{WSe}_{2}\right)$, as demonstrated in Fig. S9.

\section{CONCLUSION}

In summary, we observe that the PL intensity of monolayer $\mathrm{WS}_{2}$ can be enhanced by nearly one order of magnitude when irradiated by laser, in premise of a pre-activation PL plateau.
Atomic-scale STEM results indicate that the laser irradiation creates a large amount of sulfur vacancies, and suggest lattice oxygen substitution and related defect clustering due to the formation of oxidized compounds and nano-sized holes with oxygen-bonded edges. DFT calculations and controlled experiments further reveal that the oxygen-substituted sulfur defects and the nearly exponential dependence of their concentrations on the temperature of $\mathrm{WS}_{2}$ are the origin of the sharp PL enhancement. Our findings shed new light on the relationship between macroscopic PL property and atomic-level structures of monolayer TMDCs, which will benefit their optical related applications.

Received 24 August 2021; accepted 15 October 2021; published online 26 November 2021

1 Atkin P, Lau DWM, Zhang Q, et al. Laser exposure induced alteration of $\mathrm{WS}_{2}$ monolayers in the presence of ambient moisture. 2D Mater, 2017, 5: 015013

2 Wang QH, Kalantar-Zadeh K, Kis A, et al. Electronics and optoelectronics of two-dimensional transition metal dichalcogenides. Nat Nanotech, 2012, 7: 699-712

3 Mak KF, Shan J. Photonics and optoelectronics of 2D semiconductor transition metal dichalcogenides. Nat Photon, 2016, 10: 216-226

4 Splendiani A, Sun L, Zhang Y, et al. Emerging photoluminescence in monolayer $\mathrm{MoS}_{2}$. Nano Lett, 2010, 10: 1271-1275

5 Chhowalla M, Shin HS, Eda G, et al. The chemistry of two-dimensional layered transition metal dichalcogenide nanosheets. Nat Chem, 2013, 5: 263-275

6 Mak KF, Xiao D, Shan J. Light-valley interactions in 2D semi- 
conductors. Nat Photon, 2018, 12: 451-460

7 Mak KF, He K, Shan J, et al. Control of valley polarization in monolayer $\mathrm{MoS}_{2}$ by optical helicity. Nat Nanotech, 2012, 7: 494-498

8 Cao T, Wang G, Han W, et al. Valley-selective circular dichroism of monolayer molybdenum disulphide. Nat Commun, 2012, 3: 887

9 Li L, Shao L, Liu X, et al. Room-temperature valleytronic transistor. Nat Nanotechnol, 2020, 15: 743-749

10 Wang H, Zhang C, Rana F. Ultrafast dynamics of defect-assisted electron-hole recombination in monolayer $\mathrm{MoS}_{2}$. Nano Lett, 2015, 15: 339-345

11 Barja S, Refaely-Abramson S, Schuler B, et al. Identifying substitutional oxygen as a prolific point defect in monolayer transition metal dichalcogenides. Nat Commun, 2019, 10: 3382

12 Edelberg D, Rhodes D, Kerelsky A, et al. Approaching the intrinsic limit in transition metal diselenides via point defect control. Nano Lett, 2019, 19: 4371-4379

13 Amani M, Lien DH, Kiriya D, et al. Near-unity photoluminescence quantum yield in $\mathrm{MoS}_{2}$. Science, 2015, 350: 1065-1068

14 Kim H, Lien DH, Amani M, et al. Highly stable near-unity photoluminescence yield in monolayer $\mathrm{MoS}_{2}$ by fluoropolymer encapsulation and superacid treatment. ACS Nano, 2017, 11: 5179-5185

15 Lien DH, Uddin SZ, Yeh M, et al. Electrical suppression of all nonradiative recombination pathways in monolayer semiconductors. Science, 2019, 364: 468-471

16 Tongay S, Suh J, Ataca C, et al. Defects activated photoluminescence in two-dimensional semiconductors: Interplay between bound, charged and free excitons. Sci Rep, 2013, 3: 2657

17 Sivaram SV, Hanbicki AT, Rosenberger MR, et al. Spatially selective enhancement of photoluminescence in $\mathrm{MoS}_{2}$ by exciton-mediated adsorption and defect passivation. ACS Appl Mater Interfaces, 2019, 11: $16147-16155$

18 Zheng $\mathrm{B}$, Zheng $\mathrm{W}$, Jiang $\mathrm{Y}$, et al. $\mathrm{WO}_{3}-\mathrm{WS}_{2}$ vertical bilayer heterostructures with high photoluminescence quantum yield. J Am Chem Soc, 2019, 141: 11754-11758

19 Rosenberger MR, Chuang HJ, McCreary KM, et al. Electrical characterization of discrete defects and impact of defect density on photoluminescence in monolayer $\mathrm{WS}_{2}$. ACS Nano, 2018, 12: 1793-1800

$20 \mathrm{Hu}$ Z, Avila J, Wang X, et al. The role of oxygen atoms on excitons at the edges of monolayer $\mathrm{WS}_{2}$. Nano Lett, 2019, 19: 4641-4650

21 Kim H, Ahn GH, Cho J, et al. Synthetic $\mathrm{WSe}_{2}$ monolayers with high photoluminescence quantum yield. Sci Adv, 2019, 5: eaau4728

22 Ren J, Teng C, Cai Z, et al. Controlled one step thinning and doping of two-dimensional transition metal dichalcogenides. Sci China Mater, 2019, 62: 1837-1845

23 Nan H, Wang Z, Wang W, et al. Strong photoluminescence enhancement of $\mathrm{MoS}_{2}$ through defect engineering and oxygen bonding. ACS Nano, 2014, 8: 5738-5745

24 He Z, Wang X, Xu W, et al. Revealing defect-state photoluminescence in monolayer $\mathrm{WS}_{2}$ by cryogenic laser processing. ACS Nano, 2016, 10: 5847-5855

25 Kim MS, Yun SJ, Lee Y, et al. Biexciton emission from edges and grain boundaries of triangular $\mathrm{WS}_{2}$ monolayers. ACS Nano, 2016, 10: 23992405

26 Chen Y, Jiang Y, Yi C, et al. Efficient control of emission and carrier polarity in $\mathrm{WS}_{2}$ monolayer by indium doping. Sci China Mater, 2021, 64: 1449-1456

27 Liu H, Lu J, Ho K, et al. Fluorescence concentric triangles: A case of chemical heterogeneity in $\mathrm{WS}_{2}$ atomic monolayer. Nano Lett, 2016, 16 : 5559-5567

28 Jin C, Regan EC, Yan A, et al. Observation of moiré excitons in $\mathrm{WSe}_{2} /$ $\mathrm{WS}_{2}$ heterostructure superlattices. Nature, 2019, 567: 76-80

29 Kresse G, Furthmüller J. Efficient iterative schemes for ab initio totalenergy calculations using a plane-wave basis set. Phys Rev B, 1996, 54: 11169-11186

30 Perdew JP, Burke K, Ernzerhof M. Generalized gradient approximation made simple. Phys Rev Lett, 1996, 77: 3865-3868

31 Kresse G, Joubert D. From ultrasoft pseudopotentials to the projector augmented-wave method. Phys Rev B, 1999, 59: 1758-1775

32 Blöchl PE. Projector augmented-wave method. Phys Rev B, 1994, 50:
17953-17979

33 Zhao W, Cai S, Wei X, et al. The thinnest light disk: Rewritable data storage and encryption on $\mathrm{WS}_{2}$ monolayers. Adv Funct Mater, 2021, 31: 2103140

34 Gupta G, Majumdar K. Fundamental exciton linewidth broadening in monolayer transition metal dichalcogenides. Phys Rev B, 2019, 99: 085412

35 Huang B, Tian F, Shen Y, et al. Selective engineering of chalcogen defects in $\mathrm{MoS}_{2}$ by low-energy helium plasma. ACS Appl Mater Interfaces, 2019, 11: 24404-24411

36 Grove AS. Physics and Technology of Semiconductor Devices. 1st ed. New York: Wiley, 1967

37 Kc S, Longo RC, Wallace RM, et al. Surface oxidation energetics and kinetics on $\mathrm{MoS}_{2}$ monolayer. J Appl Phys, 2015, 117: 135301

Acknowledgements This work was supported by the National Natural Science Foundation of China (51920105002, 51991340, 51991343, and 11974156), the National Key R\&D Program of China (2018YFA0307200), Guangdong International Science Collaboration Project (2019A050510001), the Bureau of Industry and Information Technology of Shenzhen for the "2017 Graphene Manufacturing Innovation Center Project" (201901171523), Shenzhen Basic Research Project (WDZC20200819095319002, JCYJ20190809180605522, JCYJ20200109144620815, and JCYJ20200109144616617), Shenzhen Science and Technology Program (KQTD20190929173815000), the Science, Technology and Innovation Commission of Shenzhen Municipality (ZDSYS20190902092905285), and also the assistance of SUSTech Core Research Facilities, especially technical support from Pico-Centre that receives support from Presidential fund and Development and Reform Commission of Shenzhen Municipality. The calculations were supported by the fund of the Guangdong Provincial Key Laboratory of Computational Science and Material Design (2019B030301001) and also by the Introduced Innovative R\&D Team of Guangdong (2017ZT07C062 and 2019ZT08C044). All the first-principles calculations were supported by the Center for Computational Science and Engineering of Southern University of Science and Technology. We sincerely thank Prof. Feng Wang and Prof. Hui-Ming Cheng for their discussions and suggestions on this research.

Author contributions Liu B, Lin J, and Luo G conceived the project. Liu B, Lin J, and Luo G supervised the study. Zhao S prepared the samples with the help of Lai Y. Tan J and Lin J did the STEM measurements. Zhao S and Feng $S$ performed the optical measurements. Ke $\mathrm{C}$ and Luo $\mathrm{G}$ performed the theoretical analysis. Ding B helped with data analysis. All authors discussed the results and wrote the manuscript.

Conflict of interest The authors declare that they have no conflict of interest.

Supplementary information Supporting data are available in the online version of the paper.

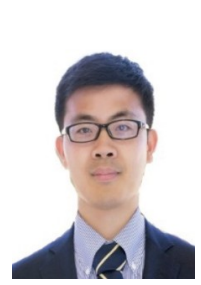

Shilong Zhao got his $\mathrm{PhD}$ degree from Tsinghua University. He is currently a postdoctoral fellow at TsinghuaBerkeley Shenzhen institute (TBSI), Tsinghua University, China. His research interests mainly focus on the properties of $2 \mathrm{D}$ materials. 


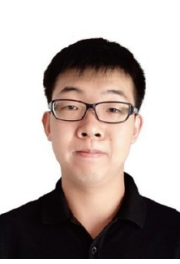

Junyang Tan received his bachelor's degree from the School of Materials Science and Engineering at Northeastern University in 2018. Currently, he is a PhD candidate under the supervision of Profs. Bilu Liu and HuiMing Cheng at TBSI, Tsinghua University. His current research interests mainly focus on the controlled preparation of $2 \mathrm{D}$ materials and their heterostructures.

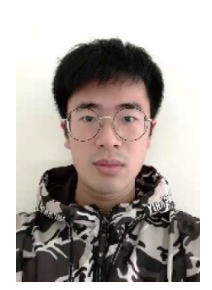

Chengxuan Ke is currently a master candidate at Southern University of Science and Technology. He received his BSc degree in electronic and information engineering at Sichuan University in 2019. His research interests focus on computational materials science based on first-principles methods.

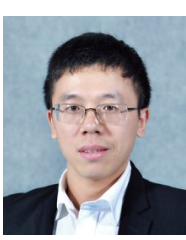

Guangfu Luo received his $\mathrm{PhD}$ degree in condensed matter physics from Peking University in 2010. Before joining Southern University of Science and Technology as a faculty member in 2018, he worked at the Institute for Molecular Science (Japan), University of Wisconsin-Madison, and Washington University in St. Louis. His research interest is computational materials design, focusing on the failure mechanisms of advanced functional materials and development of new computational methods.

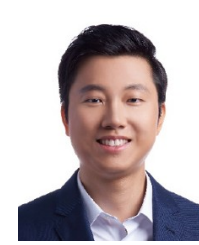

Junhao Lin got his $\mathrm{PhD}$ degree in physics from Vanderbilt University, USA, in 2015, and joined Southern University of Science and Technology (SuSTech) as an associate professor after three years of postdoctoral training at the National Advanced Institute of Science and Technology (AIST), Japan. His research interest includes the analysis of complex defect structures in novel layered materials, real-time in-situ observation of the dynamical processes in structural transition of materials under various environmental stimulations, and the phonon behavior of $2 \mathrm{D}$ materials as probed by monochromatic valence electron energy loss spectroscopy (VEELS).

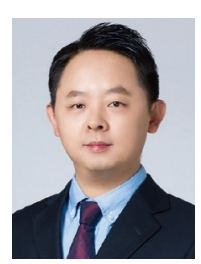

Bilu Liu is currently an associate professor and a principal investigator at TBSI, Tsinghua University, China. His research interests cover the chemistry and materials science of low-dimensional materials with an emphasis on carbon nanostructures, 2D materials, and their heterostructures. His work relates to the controlled preparation of these materials and their applications in electronics, optoelectronics, and catalysis.

\section{单层过渡金属硫族化合物中氧取代激活的光致发光 增强}

赵仕龙 ${ }^{1 \dagger}$, 谭隽阳 ${ }^{1,2 \dagger}$, 柯丞轩 ${ }^{3 \dagger}$, 冯思敏 ${ }^{1}$, 赖泳爵 ${ }^{1}$, 丁宝福 ${ }^{1}$, 罗光富 $3,4^{*}$, 林君浩 $25^{*}$, 刘碧录 $1^{*}$

摘要 具有原子层厚度的过渡金属硫族化合物 (TMDCs) 是一种适用于 光子和光电子领域的新颖的半导体材料, 而增强其光致发光 (PL)特性 对于这类材料的光学应用至关重要. 尽管研究人员做了大量的工作来 增强单层TMDCs的PL特性, 但微观结构和PL效率之间的复杂关系阻碍 了对其性质的调控. 本文中, 我们证实了在激光辐照下, 单层TMDC的 PL强度在经过一个预激活的平台后可以增强近一个数量级且半峰宽变 窄约 $20 \%$. 实验和理论研究表明, 低功率的激光辐照能够在 $\mathrm{WS}_{2}$ 中产生 许多硫空位簇, 这些硫空位族随后被氧取代并显著增强其PL. 这种PL增 强的现象在其他单层TMDC材料中有普适性, 有利于TMDC在光学领 域的应用. 